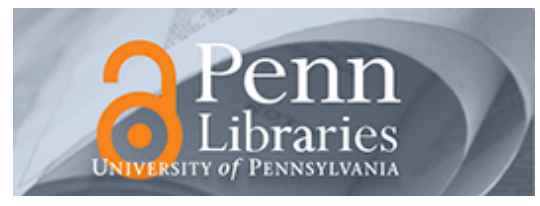

University of Pennsylvania

ScholarlyCommons

9-9-2009

\title{
Irreversible Rearrangements, Correlated Domains, and Local Structure in Aging Glasses
}

\author{
Peter Yunker \\ University of Pennsylvania, pyunker@sas.upenn.edu \\ Zexin Zhang \\ University of Pennsylvania \\ Kevin B. Aptowicz \\ West Chester University \\ Arjun G. Yodh \\ University of Pennsylvania
}

Follow this and additional works at: https://repository.upenn.edu/physics_papers

Part of the Physics Commons

\section{Recommended Citation}

Yunker, P., Zhang, Z., Aptowicz, K. B., \& Yodh, A. G. (2009). Irreversible Rearrangements, Correlated Domains, and Local Structure in Aging Glasses. Retrieved from https://repository.upenn.edu/

physics_papers/51

\section{Suggested Citation:}

Yunker, P., Zhang, Z., Aptowicz, K.B., Yodh, A.G. (2009). "Irreversible Rearrangements, Correlated Domains, and Local Structure in Aging Glasses." Physical Review Letters.103, 115701.

(C) 2009 The American Physical Society

http://dx.doi.org/10.1103/PhysRevLett.103.115701

This paper is posted at ScholarlyCommons. https://repository.upenn.edu/physics_papers/51

For more information, please contact repository@pobox.upenn.edu. 


\title{
Irreversible Rearrangements, Correlated Domains, and Local Structure in Aging Glasses
}

\author{
Abstract \\ Bidisperse colloidal suspensions of temperature-sensitive microgel spheres were quenched from liquid to \\ glass states by a rapid temperature drop, and then the glass was permitted to age. Irreversible \\ rearrangements, events that dramatically change a particle's local environment, were observed to be \\ closely related to dynamic heterogeneity. The rate of these irreversible events decreased during aging and \\ the the number of particles required to move as part of these irreversible rearrangements increased. \\ Thus, the slowing dynamics of aging were governed by growing, correlated domains of particles. \\ Additionally, short-range order developed, and a spatial decay length scale associated with orientational \\ order was found to grow during aging.

\section{Disciplines} \\ Physical Sciences and Mathematics | Physics

\section{Comments} \\ Suggested Citation: \\ Yunker, P., Zhang, Z., Aptowicz, K.B., Yodh, A.G. (2009). "Irreversible Rearrangements, Correlated Domains, \\ and Local Structure in Aging Glasses." Physical Review Letters.103, 115701. \\ (C) 2009 The American Physical Society \\ http://dx.doi.org/10.1103/PhysRevLett.103.115701
}




\title{
Irreversible Rearrangements, Correlated Domains, and Local Structure in Aging Glasses
}

\author{
Peter Yunker, ${ }^{1}$ Zexin Zhang, ${ }^{1,2}$ Kevin B. Aptowicz, ${ }^{3}$ and A. G. Yodh ${ }^{1}$ \\ ${ }^{1}$ Department of Physics and Astronomy, University of Pennsylvania, Philadelphia, Pennsylvania 19104, USA \\ ${ }^{2}$ Complex Assemblies of Soft Matter, CNRS-Rhodia-UPenn UMI 3254, Bristol, Pennsylvania 19007, USA \\ ${ }^{3}$ Department of Physics, West Chester University, West Chester, Pennsylvania 19383, USA
}

(Received 3 June 2009; revised manuscript received 3 August 2009; published 9 September 2009)

\begin{abstract}
Bidisperse colloidal suspensions of temperature-sensitive microgel spheres were quenched from liquid to glass states by a rapid temperature drop, and then the glass was permitted to age. Irreversible rearrangements, events that dramatically change a particle's local environment, were observed to be closely related to dynamic heterogeneity. The rate of these irreversible events decreased during aging and the the number of particles required to move as part of these irreversible rearrangements increased. Thus, the slowing dynamics of aging were governed by growing, correlated domains of particles. Additionally, short-range order developed, and a spatial decay length scale associated with orientational order was found to grow during aging.
\end{abstract}

DOI: 10.1103/PhysRevLett.103.115701

After initial formation, glasses relax via a nonequilibrium process called aging during which their dynamics slow dramatically and become more heterogeneous. Interestingly, diverging relaxation time scales and viscosities characteristic of the glass transition likely derive from an analogous emergence of heterogeneous particle domains that rearrange in a correlated manner [1]. The structural causes of this so-called dynamic heterogeneity, however, remain elusive. Recent work has searched for connections between dynamics and structure [2-8], for example, suggesting short-range crystalline order as a structural cause of dynamic heterogeneity $[3,4]$. The fruits of this continued search, if attained, will be directly applicable to the concepts of dynamical arrest as they apply to the glass transition and will thereby unify physical phenomena observed across a broad spectrum of jammed systems including colloidal suspensions [5], granular media [3,9], metallic glasses [6,10], and polymer glasses [11].

The slow nature of glass dynamics makes them intrinsically challenging to study, requiring observations on very short and very long time scales. In this Letter we ameliorate the time scale problem by studying the aging of colloidal glasses immediately after a deep quench [12]. Bidisperse suspensions of temperature-sensitive colloidal particles confined in two dimensions (2D) are quenched from liquid to glass states. The rapid and deep quench permits study of glass dynamics from very short time scales to very long time scales, i.e., over observation times sufficient for significant structural changes to occur and evolve. Video microscopy measurements reveal the development of shortrange order during aging and establish a direct connection between locally ordered particles and dynamic heterogeneity. We discover that most of the fast-moving particles are not major participants in the aging process. However, a careful analysis of fast particle dynamics during aging identified an increase in the cluster size of a particular class of correlated particles, clusters of fast particles par-
PACS numbers: 64.70.kj, 61.43.Fs, 64.70.pv, 82.70.Dd

ticipating in irreversible rearrangements. These irreversible rearrangements are similar to those recently identified in simulation studies [2]. The increase of irreversible rearrangement cluster size, as well as the observed increase in number of stable particle configurations, directly leads to the slowing dynamics characteristic of aging. Additionally, we find that particles with local crystalline order are very unlikely to irreversibly rearrange. Thus a direct link between local crystalline order, particle rearrangement, and slowing dynamics is experimentally demonstrated.

The experiment employs an aqueous suspension of poly( $N$-isopropyl acry-lamide) microgel colloidal spheres (i.e., NIPA particles), whose diameters increase as temperature is reduced $[13,14]$. The particles are similar to those used in recent phase transformation experiments and are described therein [15]. A binary mixture of NIPA particles (small and large diameters $D_{S}=1.09 \mu \mathrm{m}$ and $D_{L}=1.55 \mu \mathrm{m}$, respectively, at temperature $T=28.0^{\circ} \mathrm{C}$ ) is sandwiched between two glass cover slips, thereby creating a quasi-2D system. Approximately $45 \%$ (by number) of the particles are large. The softness of the NIPA colloid interparticle potential, by contrast to that of hard spheres, permits access to area fractions far above the glass transition [18].

Rapid quenching from liquid to glass is achieved by optical heating. A small amount of red dye (Chromatech-Chromatint Red 1064), 0.3\% by weight, is released into the suspension. This dye absorbs light from a mercury lamp focused through the microscope objective. In the illuminated region, the temperature is increased by $\sim 4 \mathrm{deg}$ in $\sim 0.1 \mathrm{sec}$ via light absorption and relaxation processes. The NIPA particle radii are thus abruptly decreased by $\sim 0.1 \mu \mathrm{m}$, and the local area fraction $\left(\phi_{A}\right)$ is decreased by $\sim 10 \%$. While the lamp is on, the particles are in the liquid state, as evidenced by their diffusive meansquare particle displacements (MSD): $\mathrm{MSD}=\left\langle\Delta x_{i}^{2}\right\rangle$ (see Fig. 1). When the mercury lamp is turned off, excess heat 


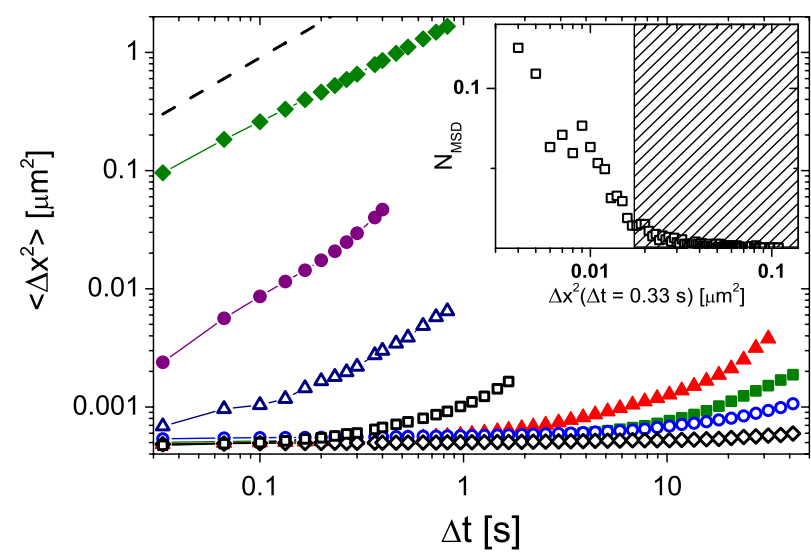

FIG. 1 (color online). Aging dynamics. Mean-square displacement at different times after the quench $\left(t_{w}\right)$ : the liquid state before the quench (solid diamonds), $t_{w}=0.25 \mathrm{~s}$ (solid circles), 1 (open triangles), 3.33 (open squares), 36 (solid triangles), 86 (solid triangles), 143 (open circles), and $10000 \mathrm{sec}$ (open diamonds). Inset: $N_{\mathrm{MSD}}$, the fraction of particles with a given mean-square displacement for $0 \leq t_{w} \leq 3 \mathrm{sec}$ and $\Delta t=$ $0.33 \mathrm{sec}$. Particles that irreversibly rearrange have mean-square displacements that fall in the shaded region. Solid lines guide the eye, and the dashed line has a slope of 1 on a log-log plot.

rapidly dissipates, and particles swell to their original size in less than $0.1 \mathrm{sec}$. The rapid change from small- $\phi_{A}$ (liquid) to large- $\phi_{A}$ creates a glass. Aging begins once the sample returns to thermal equilibrium and particles have completely returned to their original size, which is verified in situ. More information about the experimental setup and this glass forming technique is available in the online supplemental information [19] (OSI).

The data contained were collected at five final area fractions ranging from $\phi_{A}=0.81$ to $\phi_{A}=0.84$; data in Figs. 1-4 are taken solely from samples with $\phi_{A}=0.84$, where the effects are strongest (see OSI). This rapid and deep quench is a unique feature of the NIPA particle experiments which permits measurement of aging over a broad range of time scales [12] that were not accessible to previous aging experiments [20]. A similarly deep quench was reported recently by Assoud et al. [21]; their experiment used rapid magnetic field changes to quench longrange repulsive magnetic particles from liquid to glass.

During aging, glass dynamics depend on elapsed time from the quench, i.e., waiting time, $t_{w}$. The ensembleaveraged particle MSD, shown for different $t_{w}$ in Fig. 1, exhibit aging dynamics of a typical glass. The initial MSD plateau corresponds to caged particle behavior, and the "upturn" at longer times occurs when these cages rearrange. As $t_{w}$ increases, the MSD upturn occurs at later times until it finally falls outside the experimental window (see OSI [19] for a full characterization of MSD versus $t_{w}$ ).

Constituent particles in glasses tend to rearrange in a correlated manner involving many neighbors [1,5,22,23], making it difficult to determine which particle configura- tion was initially unstable by use of mean-square displacements $[24,25]$. Thus a different dynamic quantity appears to be required, one that isolates the dynamic heterogeneity essential to relaxation. In recent two-dimensional simulations, Widmer-Cooper et al. identified a class of particles that undergo irreversible rearrangements (IRs) [2]. They found that if a motional event causes a particle to lose four of its nearest neighbors, then the particle rarely recovers its initial configuration. These so-defined irreversible rearrangements facilitate differentiation between affine motions that maintain local structural configurations, and nonaffine motions that contribute to relaxation. In the present experiments, neighbors are calculated using a cutoff distance defined by the first minimum in the particle pair correlation function [26]. Defining neighbors by Voronoi tessellation gave qualitatively similar results (see OSI [19]). Within our experimental time window, particles never regain their original configurations after losing three nearest neighbors; thus we say that these particles experience an IR. These particles are among the fastest in the system (see Fig. 1 inset); less than 1\% are large spheres [27].

To study the variation in correlated rearrangements with $t_{w}$, we first determined the number of IR events occurring as a function of $t_{w}$ [Fig. 2(a)]. The rate of IRs is initially high; $\sim 90$ events occur when $t_{w}<0.4 \mathrm{sec}$. However, the rate slows dramatically thereafter, and only $\sim 15$ events occur during the remaining time. Next, we identified particles that move much farther than average. During a time period $\Delta t$, the $10 \%$ largest particle displacements were found, and these particles were identified as "fast" (this definition of fast particles follows previous experiments [5] and simulations [22,28]). Clusters of fast particles were identified by connecting nearest-neighbor pairings of fast

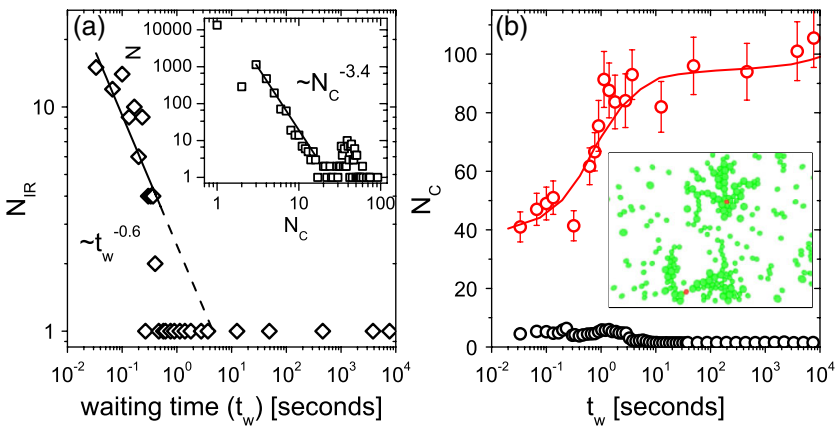

FIG. 2 (color online). Irreversible rearrangements during aging. (a) $N_{\text {IR }}$, the number of irreversible rearrangements, versus $t_{w}$. The line is a power law fit to guide the eye. Inset: Histogram of $N_{C}$, fast particle cluster sizes, for $0.03 \leq t_{w} \leq 3 \mathrm{sec}$. The line is a power law fit to guide the eye. (b) Average number of fast particles per cluster for all clusters (black circles) and the average number of fast particles per cluster for only clusters containing a particle undergoing an irreversible rearrangement (red circles), versus $t_{w}$. The line guides the eye. Inset: Snapshot of the $10 \%$ fastest particles at $t_{w}=0.62 \mathrm{sec}$, featuring two large clusters. Particles that irreversibly rearrange are plotted in red. 
particles. Since the time required for a complete rearrangement varied, we chose $\Delta t$ to maximize the average number of particles per cluster at each $t_{w}$ (see OSI [19]). The average number of particles per fast cluster versus $t_{w}$ is plotted in Fig. 2(b); it actually decreases from $\sim 5$ particles immediately after the quench, to $\sim 2$ particles at $t_{w}=$ $10000 \mathrm{sec}$. This result is surprising, as previous simulations reported a dynamic length scale that grew during aging [29].

However, closer inspection reveals that very large clusters exist, some containing $\sim 100$ particles [Fig. 2(a), inset]. Interestingly, we found that most of these large clusters contained a particle undergoing an IR. A snapshot of the $10 \%$ fastest particles, featuring two such large clusters, is shown in the inset of Fig. 2(b). We calculated the average size of these clusters of fast particles, all of which contain a particle that underwent an IR. The results are plotted in Fig. 2(b). The average size of these clusters increases from $\sim 40$ particles just after the quench, to $\sim 100$ particles at $t_{w}=10000 \mathrm{sec}$; the radius of gyration of these clusters correspondingly increases from $\sim 2.5$ to $\sim 4.5 \mu \mathrm{m}$. In other words, as the glass ages, more particles must move for IRs to occur. The observation that clusters of fast-moving particles are dramatically larger when an IR is involved demonstrates an intimate connection between IRs and dynamic heterogeneity. This effect is reminiscent of the Adam and Gibbs hypothesis, which states that as the glass transition is approached, the number of correlated particles involved in a rearrangement increases $[1,30]$. In the present case, the number of correlated fast particles involved in an irreversible rearrangement event increases with aging. Rearrangements thus become progressively more difficult to achieve, leading to slow glass dynamics and kinetic arrest (Fig. 1). At the time of the irreversible rearrangement, the cluster of fast-moving particles contains $\sim 20 \%$ of the particles that will ultimately join in the correlated domain. The remaining $\sim 80 \%$ begin to move after the irreversible rearrangement. This observation provides strong evidence that irreversible rearrangements cause the development of correlated domains of fastmoving particles. (See OSI for further discussion [19].)

Data derived from different final area fractions displayed similar behavior. The average size of a cluster containing at least one particle undergoing an IR after $t_{w}=1000 \mathrm{sec}$, increases from $\sim 70$ particles at $\phi_{A}=0.81$, to $\sim 100$ particles at $\phi_{A}=0.84$.

To better understand this growing correlated domain, we calculated the initial coordination number of particles that irreversibly rearrange, and found that $90 \%$ had $\mathrm{CN}=5$, $5 \%$ had $\mathrm{CN}=4$, and $5 \%$ had $\mathrm{CN}=6$. For comparison, at $t_{w}=10000 \mathrm{sec}, 60 \%$ of all particles have $\mathrm{CN}=6,31 \%$ have $\mathrm{CN}=5,8 \%$ have $\mathrm{CN}=7$, and $1 \%$ have $\mathrm{CN}=4$. This distribution of locally ordered stable particle configurations provides a structural source for heterogeneous dynamics in glasses [31].
In fact, the enhanced stability of locally ordered particle configurations is evident during aging. The evolution of the distribution of $\mathrm{CN}$ during aging is readily visualized by snapshots of the glass at different $t_{w}$ and is plotted in Figs. 3(a) and 3(b). Patches of particles with coordination number $\mathrm{CN}=6$, i.e., particles with local crystalline order, develop during aging, as first observed in [21].

We found that $\frac{N_{6}}{N_{\text {tot }}}$, where $N_{6}$ is the number of particles with $\mathrm{CN}=6$ and $N_{\text {tot }}$ is the total number of particles, increases from $\sim 0.48$ to $\sim 0.59$ during aging. Both large and small particles exhibit the same trend (see OSI [19]).

The spatial distribution of locally ordered configurations was studied, and clusters of particles with $\mathrm{CN}=6$ were identified by connecting nearest-neighbor pairings. We then identified the largest cluster of particles with $\mathrm{CN}=$ 6 at each $t_{w}$ [Fig. 4(b)]. At $t_{w}=0.03 \mathrm{sec}, 20 \%$ of the particles with $\mathrm{CN}=6$ are in the largest cluster, at $t_{w}=$ $0.43 \mathrm{sec}, 80 \%$ of the particles with $\mathrm{CN}=6$ are in the largest cluster and it percolates across the entire field of view $(80 \mu \mathrm{m})$, and at $t_{w}=10000 \mathrm{sec}$, almost $100 \%$ of the particles with $\mathrm{CN}=6$ are in the largest cluster. (Recall, only 15 IRs occur after $t_{w}=0.4 \mathrm{sec}$.) This large cluster of particles with $\mathrm{CN}=6$ is evident in Fig. 3(b).

The degree of local order is also characterized by the average bond orientational order parameter, $\psi_{6}=\frac{1}{N_{\mathrm{tot}} \mathrm{CN}} \times$ $\sum_{j=1}^{N_{\text {tot }}} \sum_{k=1}^{\mathrm{CN}} e^{i 6 \theta_{j k}}$. Here $\theta_{j k}$ is the angle between the $x$ axis and the $j$ - $k$ bond between particles $j$ and $k$; $\mathrm{CN}$ is the coordination number of particle $j . \psi_{6}$ is plotted in Fig. 4(c), wherein it is apparent that bond orientational order increases with $t_{w}$, consistent with previous work [3,4,21].

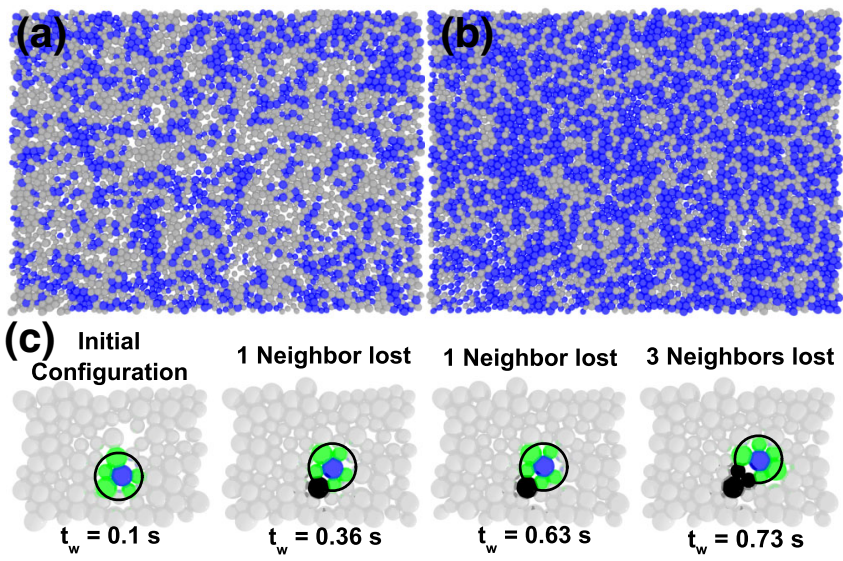

FIG. 3 (color online). Particle configuration snapshots. (a) Snapshot of colloidal glass immediately after the quench, at $t_{w}=0.03 \mathrm{~s}$. Particles with six nearest neighbors are plotted in blue. (b) Snapshot of aged colloidal glass long after the quench, at $t_{w}=10,000 \mathrm{~s}$. Particles with six nearest neighbors are plotted in blue. (c) Example of an irreversible rearrangement that finishes at $t_{w}=0.67 \mathrm{~s}$. The irreversibly rearranging particle is blue, its nearest neighbors are green, and nearest neighbors it loses are black. The circle represents the cutoff distance that defines nearest neighbors. 


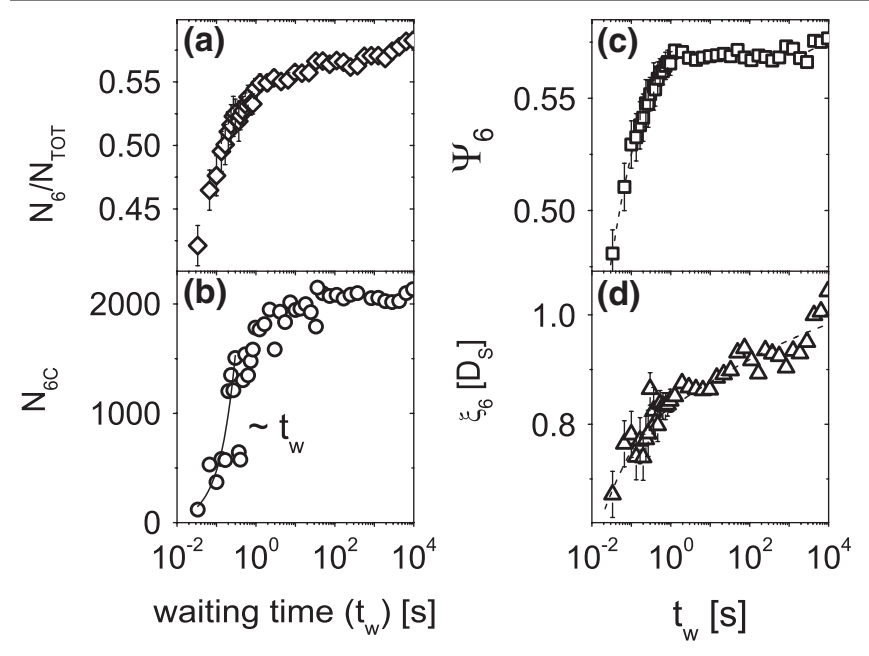

FIG. 4. Growth of local order during aging. (a) $\frac{N_{6}}{N_{\text {tou }}}$, the fraction of particles with a coordination number of 6 versus $t_{w}$. (b) $N_{6 C}$, the number of particles in the largest cluster of particles with 6 nearest neighbors versus $t_{w}$. (c) Bond orientational order parameter, $\psi_{6}$ versus $t_{w}$. (d) Orientational order correlation decay length $\xi_{6}$, extracted from $g_{6}$, versus $t_{w}$.

Correlations of $\psi_{6}$ in space are constructed, yielding the correlation function $g_{6}\left(r=\left|\mathbf{r}_{i}-\mathbf{r}_{j}\right|\right)=\left\langle\psi_{6 i}^{*}\left(r_{i}\right) \psi_{6 j}\left(r_{j}\right)\right\rangle$. We fit the envelope of $g_{6}(r)$ to an exponential $\left(e^{-r / \xi_{6}}\right)$ in order to extract a correlation decay length $\xi_{6}$ for orientational order [Fig. 4(d)] [3,4]. Initially, $\xi_{6}$ is $\sim 0.75 D_{S}$, where $D_{S}$ is the diameter of the small particles. Thus, immediately after the quench, the bond orientational order of a particle typically has minimal relation to that of its neighbor. However, after 10000 seconds, $\xi_{6}$ increased to $\sim D_{S}$. Taken together, the increase in $N_{6}, \psi_{6}$, and $\xi_{6}$ suggest that short-range order increases during aging. Thus the dramatic structural and dynamic variation that occurs during aging clearly demonstrates that particles with short-range order are more stable than particle configurations with $\mathrm{CN} \neq 6$, in agreement with Tanaka and co-workers [31], as well as with other theories that emphasize the importance of local structure $[8,33]$.

To summarize, heterogeneous glass dynamics are governed by domains of fast particles coupled to irreversible rearrangements. As a glass ages, the motion of more particles is required to accompany these irreversible rearrangement events, thus making relaxation more difficult to achieve and slowing glass dynamics. The fraction of particles with local crystalline order increases during aging. Particles with local crystalline order are especially stable and unlikely to irreversibly rearrange. These experimental results exhibit a clear connection to the slowing dynamics characteristic of aging, and to the heterogeneous dynamics of glasses.

We thank Ke Chen, Doug Durian, Wouter Ellenbrook, Piotr Habdas, Andrea Liu, Tom Lubensky, Yair Shokef, and Ning $\mathrm{Xu}$ for useful discussions. We acknowledge the financial support of the National Science Foundation through DMR-080488 (A. G. Y.) and the PENN MRSEC DMR-0520020. Z.Z. gratefully acknowledges support from Rhodia.

[1] G. Adam and J. H. Gibbs, J. Chem. Phys. 43, 139 (1965).

[2] A.Widmer-Cooper et al., Nature Phys. 4, 711 (2008).

[3] K. Watanabe and H. Tanaka, Phys. Rev. Lett. 100, 158002 (2008).

[4] T. Kawasaki, T.Araki, and H. Tanaka, Phys. Rev. Lett. 99, 215701 (2007).

[5] E. Weeks et al., Science 287, 627 (2000).

[6] Y. T. Shen et al., Phys. Rev. Lett. 102, 057801 (2009).

[7] D. B. Miracle, Nature Mater. 3, 697 (2004).

[8] G.Tarjus et al., J. Phys. Condens. Matter 17, R1143 (2005).

[9] A. R. Abate and D. J. Durian, Phys. Rev. Lett. 101, 245701 (2008).

[10] H. W. Sheng and E. Ma et al., Nature (London) 439, 419 (2006).

[11] I. M. Hodge, Science 267, 1945 (1995).

[12] W. Kob and J.-L. Barrat, Phys. Rev. Lett. 78, 4581 (1997).

[13] B. R. Saunders and B. Vincent, Adv. Colloid Interface Sci. 80, 1 (1999).

[14] R. Pelton, Adv. Colloid Interface Sci. 85, 1 (2000).

[15] A. M. Alsayed et al., Science 309, 1207 (2005); Y. Han et al., Phys. Rev. E 77, 041406 (2008); H. Senff and W. Richtering, J. Chem. Phys. 111, 1705 (1999); J. Wu, B. Zhou, and Z. Hu, Phys. Rev. Lett. 90, 048304 (2003); Y. Han et al., Nature (London) 456, 898 (2008).

[16] C. Roland et al., Rep. Prog. Phys. 68, 1405 (2005).

[17] D. Chandler and J.D. Weeks, Phys. Rev. Lett. 25, 149 (1970).

[18] Z. Zhang et al., Nature (London) 459, 230 (2009).

[19] See EPAPS Document No. E-PRLTAO-103-043938 for supplementary information. For more information on EPAPS, see http://www.aip.org/pubservs/epaps.html.

[20] G. C. Cianci, R. E. Courtland, and E. R.Weeks, Solid State Commun. 139, 599 (2006).

[21] L. Assoud et al., Phys. Rev. Lett. 102, 238301 (2009).

[22] W. Kob et al., Phys. Rev. Lett. 79, 2827 (1997).

[23] L. Berthier et al., Science 310, 1797 (2005).

[24] A. Widmer-Cooper and P. Harrowell, J. Non-Cryst. Solids 352, 5098 (2006).

[25] A. Widmer-Cooper and P. Harrowell, Phys. Rev. Lett. 96, 185701 (2006).

[26] W. Kob et al., J. Phys. Condens. Matter 12, 6385 (2000).

[27] J. M. Lynch, G. C. Cianci, and E. R. Weeks, Phys. Rev. E 78, 031410 (2008).

[28] C. Donati et al., Phys. Rev. E 60, 3107 (1999).

[29] A. Parsaeian and H. E. Castillo, Phys. Rev. E 78, 060105 (R) (2008).

[30] I. M. Hodge, Macromolecules 20, 2897 (1987).

[31] H. Tanaka, J. Non-Cryst. Solids 351, 3371 (2005).

[32] V. Ilyin et al., Phys. Rev. E 77, 061509 (2008).

[33] V. Lubchenko and P. G. Wolynes, J. Chem. Phys. 121, 2852 (2004) 